\title{
Converter placement in the interconnection of railway power grid and public power grid based on cost-reliability approach
}

\author{
Mokhtar Bozorg, Daniel Lopez, Rachid Cherkaoui \\ Power System Group \\ Ecole Polytechnique Fédérale de Lausanne (EPFL) \\ Lausanne, Switzerland \\ mokhtar.bozorg@epfl.ch
}

\begin{abstract}
The railway load in Switzerland is expected to increase in the next years. Increasing the capacity of the interconnection between the railway power system and the public grid is a reasonable solution to deal with this problem. This paper proposes a way to find the locations and capacities for new interconnections and the capacity expansion for the existing one. To show the effectiveness of the proposed model, the networks in the Suisse Romande region (French part of Switzerland) is considered as a case study.
\end{abstract}

\section{INTRODUCTION}

Two different power networks coexist nowadays in Switzerland: The Swiss power grid, which is operated by Swissgrid company and supplies most of the electricity demand in the country. It is a three-phase system that operates at a conventional frequency of $50 \mathrm{~Hz}$. The railway network is operated by SBB company which is in charge of providing energy to the railway fleet distributed over the country. This is a single-phase power system operating at $16.7 \mathrm{~Hz}$.

The railway load in Switzerland is expected to increase in the next years. This is explained by two factors: First, the aim of the railway companies to increase the frequency of commercial railway transport in order to improve the passenger service. Second, the increase of the railroad freight system.

The railway network self-generation capacity is not expected to rise in the future. This generation is mainly composed of hydropower and its expansion is limited due to the geographical and the environmental factors. Despite, the Swiss power grid generation expansion is more economic. Therefore, the interconnection capacity between these two networks will play a decisive role in the railway network to satisfy its forecasted energy demand in a few years.

Moreover, the interconnection presents great advantages for both power systems, being the most important: reliability increase, ancillary services sharing, opportunities for energy trade due to the diversity of the peak load.

From the railway system point of view the different aspects related to the operation, design and the maintenance of the railway power system are presented in [1-2]. However, the topic of the interconnection between the railway power system and the public power system with different operating frequency is not well investigated in the literatures.

From power system point of view, several literatures investigate the problem of transmission capacity expansion and substation expansion planning [3-5]. But, the problem of interconnection expansion planning between two different networks is poorly investigated.

The high cost of the elements involved in the interconnections between the railway network and the Swiss power grid makes it necessary to develop an economic analysis of the problem.

This paper proposes a way to find the optimal locations and capacities for the new interconnections and the optimal capacity expansion for the existing interconnections.

An optimization formulation is used to address the problem. During the optimization process the possible solutions, which satisfy a list of technical constraints, will be evaluated under two main criteria:

- Minimizing the investment cost of the interconnections

- Achieving the acceptable reliability level

These two concepts are often contradictory since a high reliability level is achieved by increasing the reserve capacity or reducing the component failure rate with redundancies in the system, which implies a large investment costs. This paper proposes several ways to deal with this contradiction by modifying the role of the reliability parameters within the problem formulation. 
The reliability level of a power system can be measured by means of different indexes. Two well known indexes will be used in this paper, the "loss of load probability" (LOLP) and the "expected load not served" (ELNS).

The LOLP indicates the probability that the available generation and interconnection capacity cannot cover the load of the system. It can also be seen as the portion of the time that the system will suffer from some loss of load. This index can be computed by adding the outage probabilities for every possible combination of single or simultaneous outage, only if it causes some loss of load.

The rest of this paper is organized as follows: Problem description is presented in section II. The calculation and incorporation of reliability index in the optimization problem are discussed in section III. The interconnection between the networks in "Suisse Romande" region of Switzerland is considered as a case study in section IV. Finally, the summary and future works are presented in section V.

\section{PROBLEM DESCRIPTION}

\section{A. Investment Cost}

The investment cost is dependent on the installation of new interconnections and the expansion of existing ones. The cost of installation of new interconnections involves the cost of the transmission line, which depends on the capacity and the distance between the connected substations, and the cost of converters, which depends on the capacity of the interconnection. The cost of expansion of existing interconnections is dependent on the expansion capacity. Therefore the investment cost is:

$$
\begin{array}{r}
C_{i n v}=\sum_{i j \in(i j)^{c a n}}\left(C_{i j}^{l i n e} \cdot D i s_{i j}+C_{i j}^{c o n v} \cdot \operatorname{Cap}_{i j}^{c a n}\right) \\
+\sum_{i j \in(i j)^{\text {exist }}}\left(C_{i j}^{\text {expan }} \cdot \operatorname{Cap}_{i j}^{\text {expan }}\right)
\end{array}
$$

Where $i$ and $j$ are the index for power grid substations and railway grid substations. $(i j)^{c a n}$ is the set of all candidate interconnections. $(i j)^{\text {exist }}$ is the set of all existing interconnections. Dis $s_{i j}$ is the distance between substations $i$ and $j$. $\mathrm{Cap}_{i j}^{c a n}$ is the variable representing the capacity of the candidate interconnection between substations $i$ and $j$ (0 means no interconnection). Similarly, $\mathrm{Cap}_{i j}^{\text {expan }}$ is the variable for expansion capacity of existing interconnection between substations $i$ and $j$.

$C_{i j}^{c o n v}$ is the cost of the converter between substations $i$ and $j$ in [CHF/MW]. $C_{i j}^{\text {expan }}$ is the expansion cost of existing interconnection between substations $i$ and $j$.

$C_{i j}^{l i n e}$ is the cost of the transmission line between substations $i$ and $j$ in $[\mathrm{CHF} / \mathrm{km}]$. It is assumed it that the cost of transmission line is a linear function of its capacity as formulated in (2).

$$
C_{i j}^{\text {line }}=A^{\text {line }}+B^{\text {line }} \cdot \operatorname{Cap}_{i j}^{\text {can }}
$$

Where $A^{\text {line }}$ and $B^{\text {line }}$ are the fix and variable cost function coefficients respectively.
It is difficult to estimate the cost of the components. The estimations made in this work are based on the historical financial data or market data in Switzerland.

\section{B. Technical Constraints}

The main technical constraints are the power balance for each substation of the system, the voltage level constraints for each interconnection and the power flow limitation of each transmission line. The constraints are presented from the railway system point of view as follows.

1) Power Balance:

The power balance for each bus of the railway system has to be guaranteed. It is defined by the following equation:

$$
\sum_{i} F_{i j}+G_{j}-D_{j}-\sum_{j^{\prime}} F_{j j^{\prime}}=0 \quad \forall j
$$

Where $G_{j}$ and $D_{j}$ are the generation and the load in substation $j . F_{j j}$ is the power flow from substation $j$ to $j^{\prime}$ of the railway grid. $F_{i j}$ is the power flow from substation $i$ of the power grid to substation $j$ of the railway grid.

2) Voltage Level:

The maximum and minimum capacity of each candidate interconnection depends on the level of the voltage and the size of the corresponding substation in the power grid. The power grid substations have been aggregated by their voltage level in three groups, depending on their appropriate interconnection capacity range. Therefore, the voltage level constraints can be expressed as follows:

$$
\begin{gathered}
\operatorname{Cap}_{i j}^{c a n}<\operatorname{Cap}^{1} \quad i \in S_{1}^{V} \\
\operatorname{Cap}^{1}<\operatorname{Cap}_{i j}^{c a n}<\operatorname{Cap}^{2} \quad i \in S_{2}^{V} \\
\operatorname{Cap}_{i j}^{c a n}>\operatorname{Cap}^{3} \quad i \in S_{3}^{V}
\end{gathered}
$$

$S_{k}^{V}$ is the set of all substations with capacity limitation lower than $\operatorname{Cap}^{k}$.

3) Power Flow:

The location and the capacity of the interconnections must ensure the transmission of energy to the load points, avoiding any line congestion during a normal operation state. This implies that the power flow through each line of the railway system must be less than the maximum transmission capacity of the line. Also, the power flow between two networks in each interconnection is limited to the capacity of the interconnection.

$$
\begin{aligned}
& F_{j j^{\prime}} \leq F_{j j^{\prime}}^{\max } \\
& F_{i j} \leq \operatorname{Cap}_{i j}^{c a n} \\
& F_{i j} \leq \operatorname{Cap}_{i j}^{\text {expan }}+\operatorname{Cap}_{i j}^{\text {exist }} \\
& i j \in(i j)^{c a n} \\
& i j \in(i j)^{\text {exist }}
\end{aligned}
$$

The power flows over the railway network can be estimated by solving an optimal DC load flow. The power injected at each bus is then obtained by adding the generation in that bus, the power flow coming from the power grid (if an interconnection is scheduled) and subtracting the load located in the bus. 
An optimal load flow consists of solving an optimization problem where the constraints are given by the DC load flow equations and the objective function represents the total cost associated with the power injected into the system. This procedure will provide a way to find the power exchanged through the interconnections in an optimal operation state and will allow us to ensure that all the transmission lines operate within their acceptable capacity range.

\section{Reliability Criteria}

There are two approaches to include the reliability criteria in the optimization model.

The first approach introduces a new constraint in the problem formulation that sets a minimum acceptable reliability level. This level can be defined using the both reliability indexes. The ELNS provides a better idea of the system reliability since it not only considers the loss of load probability resulting from the different outages, but also the amount of load curtailed as a consequence of those outages.

$$
E L N S \leq E L N S^{\text {desired }}
$$

The second approach presents a way to find a balance between the investment cost and the reliability level. This is done by considering both issues in the objective function: a cost is associated to each expected megawatt not served and this cost is added to the investment cost in the updated function.

$$
\text { Minimize } F=C_{i n v}+C_{E L N S}
$$

In order to be able to compare both costs, the $C_{E L N S}$ has to be defined as an operating cost, i.e. it must be evaluated over the amortization time $(\mathrm{T})$ of the new equipment:

$$
C_{E L N S}=\sum_{t=1}^{T} E L N S(t) \cdot U C
$$

Where $U C$ is the estimated unitary cost of load not served in $[\mathrm{CHF} / \mathrm{MW}]$.

It is important to remark that the $U C$ must consider not only the energy price but also the consequences that a lack of supply has on the demand.

As it happened in the previous approach, it is possible to include in the formulation the demand features of the power system (i.e. The sensitivity of the demand against a loss of load) by modifying the value of the $U C$. The advantage of this approach is that it evaluates the two main issues of the problem and finds the best solution that balances both costs.

\section{RELIABILITY INDEX CALCULATIONS}

The reliability of the system can be checked by its answer to the following question: when a contingency occurs, are the remaining elements able to handle the new situation in order to satisfy the loads? In this paper to calculate the reliability index for the railway system, the availability of generators and interconnections are considered.

This model considers the system as a generation point that includes the generator and the interconnection capacity and a load point which contains the sum of all the loads of the system.

The probability of the outage of a single element of the system (generator or interconnection) can be calculated as the probability of that element being unavailable times the rest of the elements being available:

$$
\Phi_{k}^{1}=U_{k} \cdot \prod_{\substack{r=1 \\ r \neq k}}^{n} A_{r}
$$

Where $A$ and $U$ are the availability and unavailability of a certain element. The next reasoning can be used to calculate the probability of outage of two elements:

$$
\Phi_{k w}^{2}=U_{k} \cdot U_{w} \cdot \prod_{\substack{r=1 \\ r \neq k, w}}^{n} A_{r}
$$

The previous equations are only valid if we know exactly the elements that constitute our system. Since the purpose of the problem is to determine the optimal number and capacity of interconnections, we can generalize these equations to include every possible interconnection. This requires the addition of a set of binary variables $u_{k}(k=1,2, \ldots, n)$ associated to every possible element of the system, i.e. all the generators and all the possible interconnections between the power grid and the railway network. These variables will take the value ' 1 ' if the element $k$ is finally scheduled in the optimal solution and ' 0 ' otherwise. In other words, they will be 1 for all the generators (since they are already part of the system) and 1 or 0 for the possible interconnections depending on whether they are included or not in the optimal solution.

The probability of a single outage can then be expressed as in (15):

$$
\begin{gathered}
\Phi_{k}^{1}=u_{k} U_{k} \cdot \prod_{\substack{r=1 \\
r \neq k}}^{n}\left(1-u_{r} U_{r}\right) \\
\Phi_{k w}^{2}=u_{k} U_{k} u_{w} U_{w} \cdot \prod_{\substack{r=1 \\
r \neq k, w}}^{n}\left(1-u_{r} U_{r}\right)
\end{gathered}
$$

The same reasoning can be used to calculate the probabilities of the simultaneous outages of two components as in (16) or even more than two outages. However, these situations are so unlikely that their probabilities can be neglected without a significant loss of accuracy.

The outage of certain elements of the system may be acceptable as long as the rest can wholly supply the load without loss of continuity (i.e. it does not cause a loss of load). Consequently, in order to assess the reliability level of the system, a new set of binary variables $\sigma_{k}(k=1,2, \ldots, n)$ is included. They indicate whether the outage of element $k$ causes a loss of load (' 1 ') or not (' 0 ').

These variables can be modeled by the following linear inequalities (17): 


$$
\frac{L-\sum_{\substack{r=1 \\ r \neq k}}^{n} P_{r}}{\sum_{r=1}^{n} P_{r}} \leq \sigma_{k} \leq 1+\frac{L-\sum_{\substack{r=1 \\ r \neq k}}^{n} P_{r}}{\sum_{r=1}^{n} P_{r}}
$$

Where $L$ represents the total load of the system and $P_{r}$ is the capacity of the element $r$. From the previous expression, when element $k$ is out, if the rest of the elements can cover the load ( $\sum_{\substack{r=1 \\ r \neq k}}^{n} P_{r}>L$ ), the lower boundary of $\sigma_{k}$ will be relaxed but the upper boundary will be lower than one; hence, $\sigma_{k}$ will be ' 0 '. Otherwise, if the capacity remaining cannot cover the load ( $\sum_{\substack{r=1 \\ r \neq k}}^{n} P_{r}<L$ ), the lower boundary will be greater than zero and the upper boundary will be relaxed; hence, $\sigma_{k}$ will be ' 1 '.

Since a double outage has also been considered in this study, a second set of binary variables must be defined in order to indicate the presence of the loss of load caused by a double outage. They are named $\sigma_{k w}(k, w=1,2, \ldots, n)$ and are modeled as follows:

$$
\frac{L-\sum_{\substack{r=1 \\ r \neq k, w}}^{n} P_{r}}{\sum_{r=1}^{n} P_{r}} \leq \sigma_{k w} \leq 1+\frac{L-\sum_{\substack{r=1 \\ r \neq k, w}}^{n} P_{r}}{\sum_{r=1}^{n} P_{r}}
$$

LOLP index can be computed by adding the outage probabilities for every possible combination of single or simultaneous outages, only if it causes some loss of load. The formulation is the following:

$$
L O L P=\sum_{k=1}^{n} \sigma_{k} \Phi_{k}^{1}+\sum_{k=1}^{n} \sum_{w>k}^{n} \sigma_{k w} \Phi_{k w}^{2}+\cdots
$$

The ELNS can be obtained by multiplying the probability of every single or multi-outage state by the loss of load caused by the outage.

$$
\begin{aligned}
\text { ELNS }=\sum_{k=1}^{n} \sigma_{k} \Phi_{k}^{1} & \cdot\left(L-\sum_{\substack{r=1 \\
r \neq k}}^{n} P_{r}\right) \\
& +\sum_{k=1}^{n} \sum_{w>k}^{n} \sigma_{k w} \Phi_{k w}^{2} \\
& \cdot\left(L-\sum_{\substack{r=1 \\
r \neq k, w}}^{n} P_{r}\right)+\cdots
\end{aligned}
$$

These two metrics provide useful information about the reliability level of the system. However, the expressions above present one obstacle from a computational point of view; they are not linear with respect to the variables of the problem $\left(\sigma_{k}, \sigma_{k w}, u_{k}, P_{r}\right)$. This obstacle can be solved by linearizing the equations. The linearization technique is presented more in detail in the Appendix.

The load of the system is highly fluctuating in the time. There are many parameters that have influence over the load level. Therefore, in order to improve the accuracy of the proposed method, the load also has to be considered in a probabilistic way. This means that the load does not have a deterministic value and it can be represented by a discrete probability distribution function.

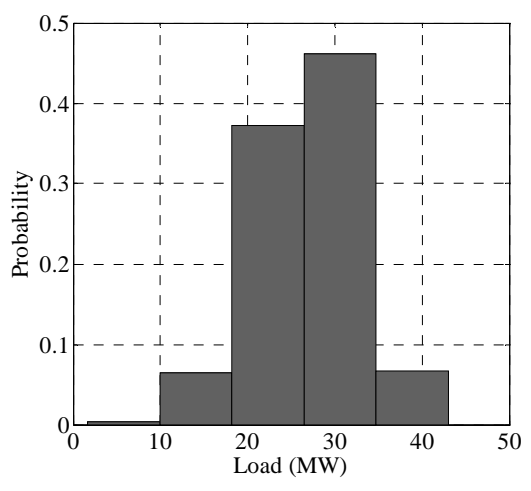

Figure 1. Load probability function with 5 intervals in substation R_4

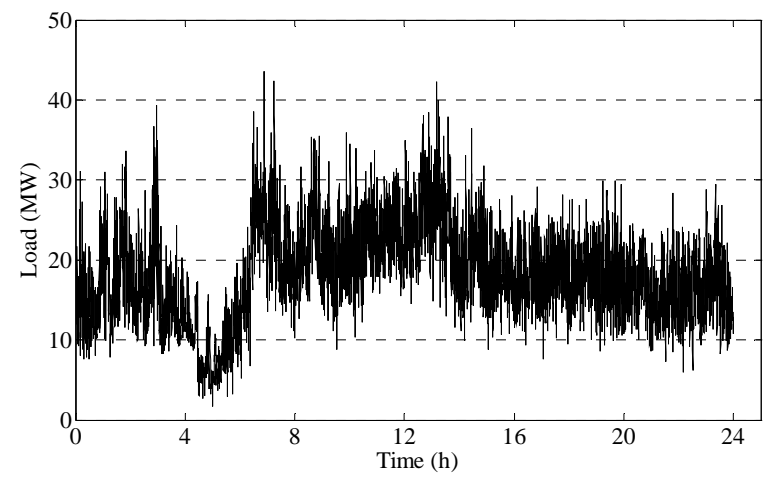

Figure 2. Daily Load variation of substation R_4 in 01.09.2012

$$
\left\{\begin{array}{c}
\text { Probability }\left(L^{l-1}<L \leq L^{l}\right)=\rho^{l} \\
\sum_{l=1}^{N_{L}} \rho^{l}=1
\end{array}\right.
$$

Where $N_{L}$ is the total number of the load levels. For each load level $\left(L^{l}\right)$ the value of reliability indexes $L O L P^{l}$ and $E L N S^{l}$ could be found using the equations (15-20). Then, the values of reliability indexes of the system considering the load variations are as follows:

$$
\begin{aligned}
& E L N S=\sum_{l=1}^{N_{L}} \rho^{l} E L N S^{l} \\
& L O L P=\sum_{l=1}^{N_{L}} \rho^{l} L O L P^{l}
\end{aligned}
$$

\section{CASE STUDY}

The "Suisse Romande" region of Switzerland is considered a case study. The railway system in this region has 11 substations (132 KV), 12 transmission lines and 2 generators. The maximum load of the substations in the railway system is presented in Table I. The hydropower generators are connected to R_1 (92.6MW) and R_2 (107MW). Five load levels are considered to model the load 
variations for each substation. Figure I shows the daily load curve of the substation R_4. The load variation in this substation for a given day in 2012 is also depicted in Figure II.

The public grid in this region includes 20 substations at $220 \mathrm{KV}$ and 2 substations at $380 \mathrm{KV}, 29$ transmission lines and 6 generators.

It can be seen in Table I that the load of the railway system in this region is less than its generation. The aim of this study is to find the size and the location of new interconnection between railway system and public power grid to cover the load with minimum investment cost. This cost minimization is under technical constraints and reliability constraint as presented in equations (3-9) and equation (10), respectively. Moreover, to find the reliability indexes, the equations (15-20) and (22-23) have to be considered as equality and inequality constraints in the minimization problem.

The economic parameters (cost function coefficients) and the reliability parameters (unavailabilities of the converters and generators) of the problem are presented in Table II. Also, the minimum capacity of the candidate interconnection to connect to the $380 \mathrm{KV}$ substation is assumed to be $25 \mathrm{MW}$. The geographic distribution of the substations in two networks is depicted in figure 3 . The distances between the substations of two networks vary between $2.7 \mathrm{Km}$ and $106 \mathrm{Km}$. It is also assumed that the public power grid is able to energize the converters up to $100 \mathrm{MW}$.

The linearized optimization problem has been implemented in the GAMS environment. Table III shows the optimum location and the optimum size of the new converters while two desired values for ELNS of the railway system have been considered. The investment costs for ELNS $S^{\text {desired }}$ equal to 0.1 and 0.01 are 11.3 MCHF and 15.3 MCHF respectively.

The solution of the optimization problem depends on the desired value of reliability indexes. Therefore, a sensitivity analysis has been done to investigate it. Figure 4 and figure 5 show the change in the minimum investment cost and total capacity of new converter by changing the desired value of ELNS. Not surprisingly, both figures indicating that the high total size and the consequent high investment cost required to achieve high reliability level.

\section{SUMMARY AND FUTURE WORKS}

The study presented in this paper proposed a methodology to assess the optimal interconnection configuration between two power systems: the Swiss Power Grid and the Swiss railway network. The cost-reliability approach has been used to address the problem. This has allowed us to provide the company with a set of optimal solutions according to the different criteria.

There are several complementary studies to improve this optimization approach that the authors will consider in their future work. The most important one is that, the optimization problem is static. A dynamic optimization considering the operation cost and load variation over a time horizon should be proposed.
TABLE I. MAXIMUM LOAD OF RAILWAY SYSTEM SUBSTATIONS

\begin{tabular}{|c|c|c|c|c|c|c|}
\hline Substation & R_1 & R_2 & R_3 & R_4 & R_5 & R_6 \\
\hline$L_{\text {max }}(\mathrm{MW})$ & 43.6 & 50 & 30 & 43.6 & 21.8 & 21.8 \\
\hline Substation & R_7 & R_8 & R_9 & R_10 & R_11 & All \\
\hline$L_{\text {max }}(\mathrm{MW})$ & 15 & 21.8 & 21.8 & 30 & 10.9 & 310.3 \\
\hline
\end{tabular}

TABLE II. COST AND RELIABILITY DATA

\begin{tabular}{|c|c|c|c|c|}
\hline$C_{i j}^{\text {conv }}\left(\frac{\mathrm{CHF}}{\mathrm{MW}}\right)$ & $A^{\text {line }}\left(\frac{\mathrm{CHF}}{\mathrm{Km}}\right)$ & $B^{\text {line }}\left(\frac{\mathrm{CHF}}{\mathrm{MW} \mathrm{Km}}\right)$ & $U^{\text {conv }}$ & $U^{\text {gen }}$ \\
\hline 737500 & 475000 & 250 & 0.02 & 0.05 \\
\hline
\end{tabular}

TABLE III. THE OPTIMUM LOCATION AND SIZE OF NEW CONVERTERS

\begin{tabular}{|c|c|c|c|}
\hline \multirow{2}{*}{ ELNS desired } & \multicolumn{2}{|c|}{ Converter location } & \multirow{2}{*}{$\begin{array}{c}\text { Size } \\
\text { (MW) }\end{array}$} \\
\cline { 2 - 3 } & $\begin{array}{c}\text { From: Public } \\
\text { grid substation }\end{array}$ & $\begin{array}{c}\text { To: Railway } \\
\text { grid substation }\end{array}$ & \\
\hline \multirow{3}{*}{$0.1 \mathrm{MW}$} & N_18 & R_2 & 74.1 \\
\cline { 2 - 4 } & N_10 & R_10 & 36.6 \\
\cline { 2 - 4 } & N_8 & R_6 & 36.6 \\
\hline \multirow{3}{*}{0.01 MW } & N_18 & R_2 & 20.9 \\
\cline { 2 - 4 } & N_2 & R_1 & 89.8 \\
\cline { 2 - 4 } & N_8 & R_6 & 89.8 \\
\hline
\end{tabular}

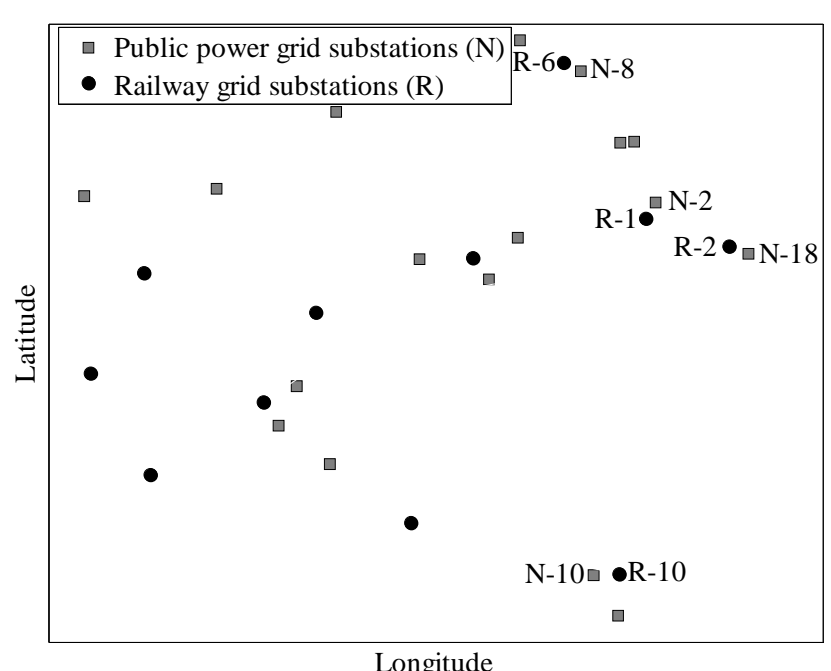

Figure 3. Location of the substations 


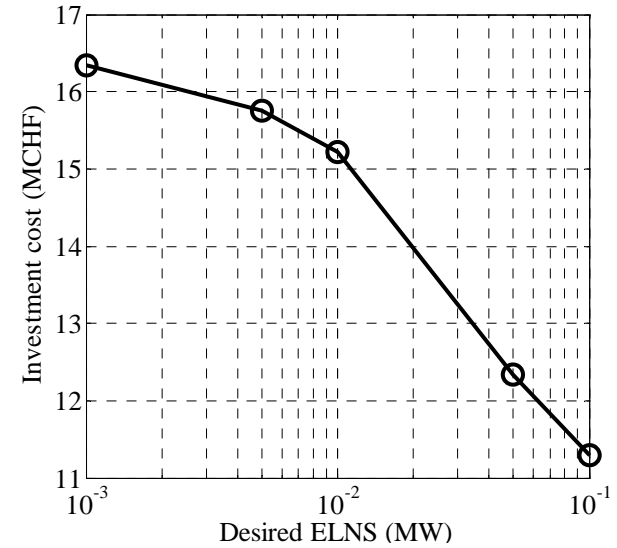

Figure 4. Minimum Investment cost

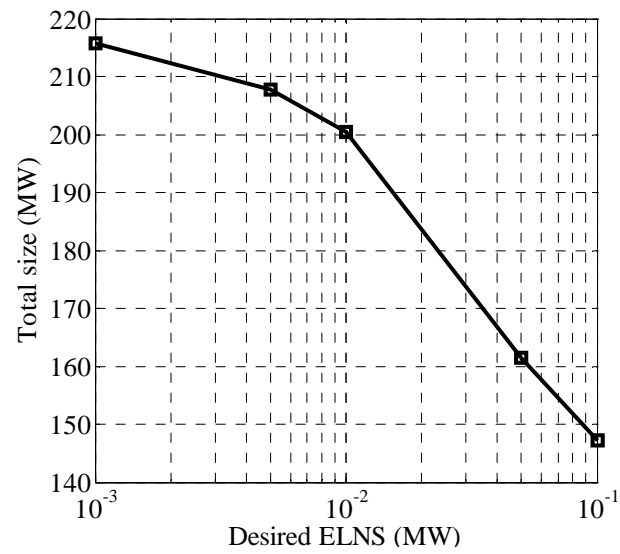

Figure 5. Total size of the new converters

\section{APPENDIX: LINEARIZATION TECHNIQUE}

The linearization technique presented in [6] and [7] is used in our proposed model 1) to transform a product of binary variables into a set of additional inequalities with a new continuous variable, and 2) to express the bilinear product of a binary variable and a continuous variable in as a set of additional linear constraints.

Let $x$ be the product of $n$ binary variables $u_{1}, \ldots, u_{n}$. Assuming that $x$ is a continuous variable, this product $\left(x=\prod_{i=1}^{n} u_{i}\right)$ is equivalent to the $n+2$ following linear inequalities:

$$
\begin{gathered}
x \geq 0 \\
x \leq u_{i} \quad i=1, \ldots, n \\
x \geq \sum_{i=1}^{n} u_{i}-n+1
\end{gathered}
$$

Generally, the basic idea for modeling the bilinear product of a bounded continuous variable $y \in\left[y^{\min }, y^{\max }\right]$ and $0-1$ variable $x$ is to introduce a new continuous variable $h$ such that $h=x y$. This product is equivalent to the additional linear constraints as follows:

$$
\begin{gathered}
x y^{\min } \leq h \leq x y^{\max } \\
y-y^{\max }(1-x) \leq h \leq y-y^{\min }(1-x)
\end{gathered}
$$

Note that if $x=0$, then the first constraint (27) implies that $h=0$,while the second constraint (28) is relaxed. Otherwise, when $x=1$, the second constraint implies that $h=y$, while the first constraint is inactive.

\section{REFERENCES}

[1] A. Steimel, Electric traction-motive power and energy supply, Oldenbourg Industrieverlag, 2008.

[2] T. Murthy, J. Korber and B. Mellitt, Computers in railway operations, Computational mechanics publications springer, 1987.

[3] Ramirez-Rosado, I. J.; Bernal-Agustin, J. L.; , "Reliability and Costs Optimization for Distribution Networks Expansion Using an Evolutionary Algorithm," Power Engineering Review, IEEE, vol.21, no.4, pp.70, April 2001

[4] Sepasian, M.S.; Seifi, H.; Foroud, A.A.; Hosseini, S.H.; Kabir, E.M.; "A new approach for substation expansion planning," Power Systems, IEEE Transactions on , vol.21, no.2, pp. 997- 1004, May 2006.

[5] Carrano, E.G.; Takahashi, R.H.C.; Cardoso, E.P.; Saldanha, R.R.; Neto, O.M.; , "Optimal substation location and energy distribution network design using a hybrid GA-BFGS algorithm," Generation, Transmission and Distribution, IEE Proceedings- , vol.152, no.6, pp. 919- 926, 4 Nov. 2005.

[6] H. P. Williams, Model Building in Mathematical Programming. New York, NY, USA: Wiley, 1999.

[7] C. A. Floudas, Nonlinear and Mixed-Integer Optimization: Fundamentals and Applications. New York, NY, USA: Oxford Univ. Press, 1995. 